\title{
Patched 1 Expression Correlates with Biochemical Relapse in High-Risk Prostate Cancer Patients
}

\author{
Annelies Gonnissen, ${ }^{* \dagger}$ Sofie Isebaert, ${ }^{* \dagger}$ Christiaan Perneel, ${ }^{\ddagger}$ Chad M. McKee,${ }^{\S}$ Filip Van Utterbeeck, ${ }^{\ddagger}$ Evelyne Lerut, ${ }^{\natural}$
} Clare Verrill, Richard J. Bryant, "Steven Joniau, ${ }^{* *}$ Ruth J. Muschel, ${ }^{\S}$ and Karin Haustermans ${ }^{* \dagger}$

\begin{abstract}
From the Laboratory of Experimental Radiotherapy, * Department of Oncology, KU Leuven - University of Leuven, Leuven; the Departments of Radiation Oncology, ${ }^{\dagger}$ Pathology, ${ }^{\lceil}$and Urology, ${ }^{* *}$ University Hospitals Leuven, Leuven, Belgium; the Department of Applied Mathematics, ${ }^{\ddagger}$ Royal Military Academy, Brussels, Belgium; the Department of Oncology, ${ }^{\S}$ Cancer Research UK/Medical Research Council Oxford Institute for Radiation Oncology, and the Nuffield Department of Surgical Sciences," John Radcliffe Hospital, University of Oxford, Oxford, United Kingdom
\end{abstract}

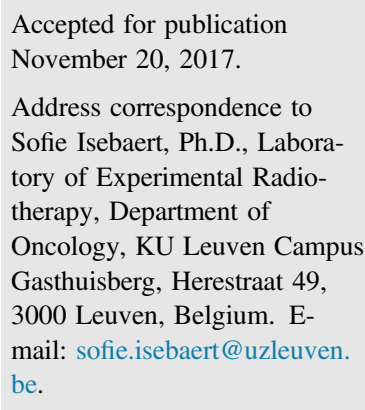

Address correspondence to Sofie Isebaert, Ph.D., Laboratory of Experimental Radiotherapy, Department of Oncology, KU Leuven Campus Gasthuisberg, Herestraat 49, 3000 Leuven, Belgium. Email: sofie.isebaert@uzleuven. be.

\begin{abstract}
There is an unmet clinical need for adequate biomarkers to aid risk stratification and management of prostate cancer (PCa) patients. Even within the high-risk PCa category, not all patients will invariably have a poor prognosis, and improved stratification of this heterogeneous group is needed. In this context, components of the hedgehog $(\mathrm{Hh})$ pathway may have promise as biomarkers, because the available evidence suggests increased Hh pathway activity may confer a poorer outcome in advanced and castrate-resistant PCa. In this study, potential associations between Hh pathway protein expression and clinicopathological factors, including time to biochemical recurrence (BCR), were investigated using a tissue microarray constructed from benign and malignant prostate samples from 75 predominantly high-risk PCa patients who underwent radical prostatectomy. Hh signaling activity was found to differ between benign and malignant prostate tissue, with a greater amount of active Hh signaling present in malignant than benign prostate epithelium. High expression of Patched 1 in malignant prostate epithelium was found to be an independent predictor of BCR in high-risk PCa patients. Gliomaassociated oncogene 1 may potentially represent a clinically useful biomarker of an aggressive tumor phenotype. Evaluation of Hh signaling activity in PCa patients may be useful for risk stratification, and epithelial Patched 1 expression, in particular, may be a prognostic marker for BCR in high-risk PCa patients. (Am J Pathol 2018, 188: 795-804; https://doi.org/10.1016/j.ajpath.2017.11.019)
\end{abstract}

Prostate cancer (PCa) is the most common solid organ malignancy diagnosed in men in developed countries, and it is the second leading cause of male cancer-related death. ${ }^{1}$ Patients with $\mathrm{PCa}$ who will potentially receive radical treatment [radical prostatectomy (RP) or radical radiotherapy] are currently stratified as having low-, intermediate-, or high-risk disease based on the risk of post-treatment relapse, and these risk categories use clinicopathological factors, including prostate-specific antigen level, clinical $\mathrm{T}$ stage, and biopsy Gleason sum score (GS). ${ }^{2,3}$ However, even within the different risk categories, a wide range of clinical outcomes of treatment exist. At the present time, clinicians do not routinely evaluate the underlying molecular features of $\mathrm{PCa}$ that might promote increased tumor aggressiveness, or use this information in decision making, and this is partly attributable to a lack of robust clinically applicable biomarkers of aggressive disease. ${ }^{4,5}$ Robust biological markers of potentially aggressive disease are therefore necessary to improve the current prognostic groupings and better identify patients most likely to benefit

\footnotetext{
Supported by the National Cancer Plan Action 29 Belgium grant KPC_29_023 (A.G. and S.I.); the Confidence in Concept of the Medical Research Council at University of Oxford and Cancer Research UK (C.M.M. and R.J.M.); the National Institute for Health Research, Cancer Research UK and the Academy of Medical Sciences (R.J.B.); and the National Institute for Health Research Oxford Biomedical Research Centre based at the Oxford University Hospitals National Health Service Trust and the University of Oxford (C.V.). K.H. is a clinical research fellow of the Research Foundation Flanders.

A.G. and S.I. contributed equally to this work.

Disclosures: None declared.
} 


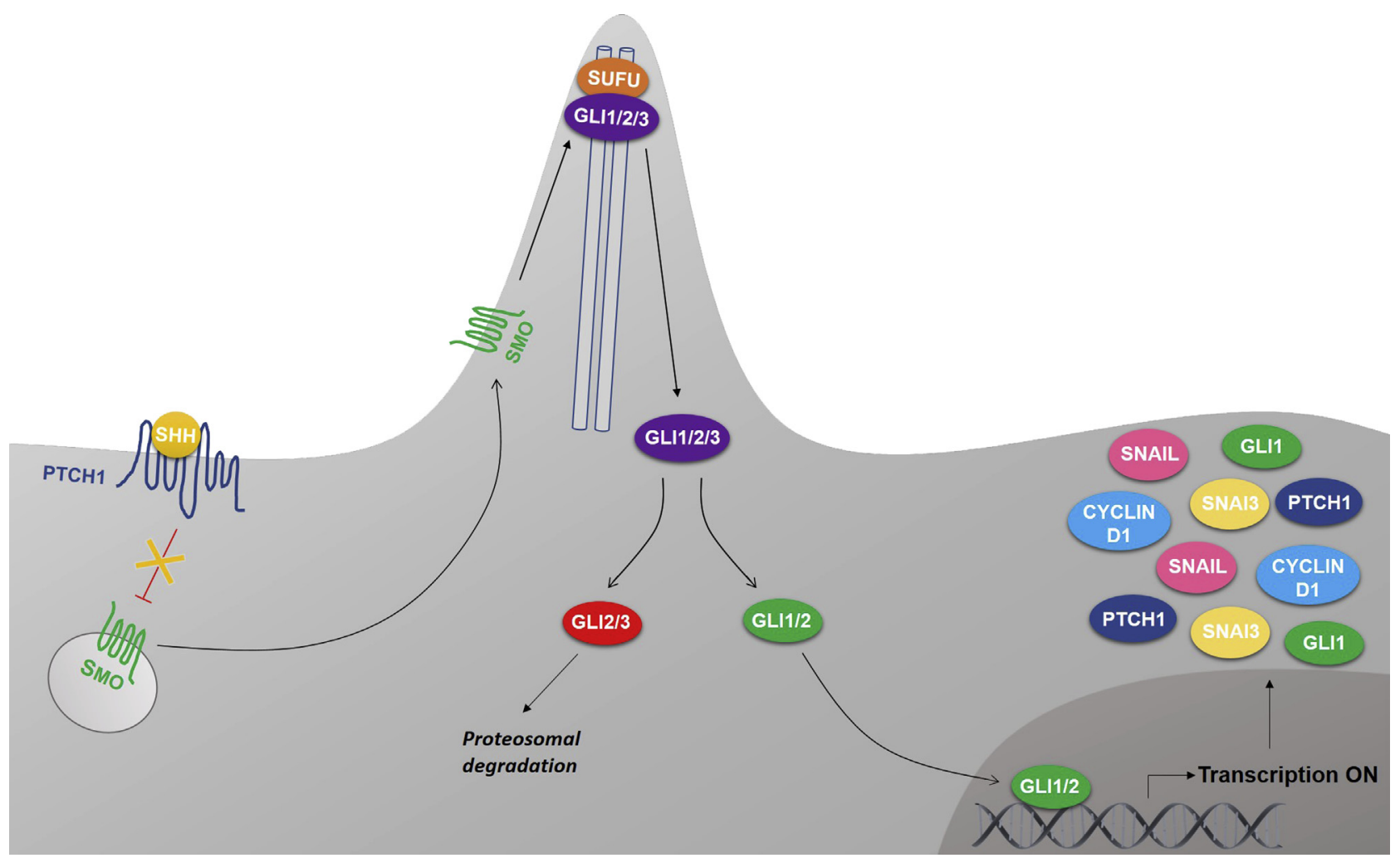

Figure 1 Schematic illustration of the main hedgehog $(\mathrm{Hh})$ pathway components. The Hh pathway is activated when Sonic Hh ligand binds to the Patched 1 (PTCH1) membrane receptor, thereby relieving the inhibitory effect of PTCH1 on Smoothened (SM0). Activated SM0 is released into the cytoplasm and accumulates in the primary cilium, which, in turn, facilitates the dissociation of the glioma-associated oncogene (GLI) transcription factors from suppressor of fused (SUFU). The GLI transcription activators translocate to the nucleus, where they promote the transcription of Hh target genes, including GLI1, PTCH1, snail family transcriptional repressor 1 (SNAIL), snail family transcriptional repressor 3 (SNAI3), and cyclin D1.

from radical treatment based on their high risk of otherwise developing systemic or lethal disease.

Previous studies indicate that elevated hedgehog (Hh) signaling pathway activity occurs in advanced and androgenresistant PCa. ${ }^{6-12}$ We hypothesize that increased Hh signaling pathway activity may represent a promising biomarker of increased disease aggressiveness in high-risk PCa patients receiving RP with curative intent. For this patient group in particular, further risk stratification is warranted because these patients have the highest risk of biochemical failure, metastatic progression, and eventual PCa-related death, but large variations in outcome are still notable within this group. ${ }^{5,1}$

Aberrant $\mathrm{Hh}$ signaling in $\mathrm{PCa}$ is thought to be ligand dependent; however, it remains unclear whether this is mediated in a paracrine and/or autocrine manner. ${ }^{8}$ The $\mathrm{Hh}$ signaling cascade (Figure 1) is activated when Sonic $\mathrm{Hh}(\mathrm{SHH})$ ligand binds to the Patched 1 (PTCH1) membrane receptor. PTCH1 is a negative regulator of $\mathrm{Hh}$ signaling and inhibits activation of the downstream target Smoothened (SMO). Binding of SHH to PTCH1 overcomes this inhibition, releasing SMO into the cytoplasm. This activates the glioma-associated oncogene (GLI) transcription factors by facilitating their dissociation from suppressor of fused (SUFU). ${ }^{14,15}$ The GLI transcription factor family consists of three members (GLI1, GLI2, and GLI3). GLI1 is the primary transcriptional activator, whereas
GLI2 and GLI3 can have activator or repressor function, depending on cellular context. ${ }^{16-22}$

To investigate the potential clinical utility of evaluating the expression level of key Hh signaling components in a cohort of predominantly high-risk localized $\mathrm{PCa}$, Hh protein expression in malignant and surrounding benign prostate tissue was compared in a tissue microarray. To investigate the mode of Hh signaling in $\mathrm{PCa}$ and surrounding benign tissue, potential correlations between the expression levels of different $\mathrm{Hh}$ proteins were assessed. The clinical relevance of $\mathrm{Hh}$ signaling in localized high-risk $\mathrm{PCa}$ was evaluated through potential associations between $\mathrm{Hh}$ protein expression and clinicopathological factors, and the prognostic value of Hh signaling was evaluated.

\section{Materials and Methods}

\section{Tissue Microarray Construction}

Tissue microarrays were constructed from 450 samples of whole-mount formalin-fixed, paraffin-embedded specimens from $75 \mathrm{RP}$ patients from KU Leuven, ${ }^{23}$ with four cores taken from the index tumor, and two cores taken from benign tissue, to account for potential intratumor heterogeneity. Full clinicopathological and outcome data 
Table 1 Patient and Tumor Characteristics $(n=75)$

\begin{tabular}{|c|c|}
\hline Characteristics & Values \\
\hline Median age at surgery, years (range) & $66(49-74)$ \\
\hline Median PSA level, ng/mL (range) & $10.4(1.5-70.9)$ \\
\hline \multicolumn{2}{|l|}{ Clinical T stage } \\
\hline$\leq 1 \mathrm{c}$ & $1(1.3)$ \\
\hline $2 a-2 c$ & $19(25.3)$ \\
\hline$\geq 3 a$ & $55(73.3)$ \\
\hline \multicolumn{2}{|l|}{ Biopsy Gleason score } \\
\hline$\leq 6(3+3)$ & $3(4.0)$ \\
\hline $7(3+4)$ & $23(30.7)$ \\
\hline $7(4+3)$ & $16(21.3)$ \\
\hline$\geq 8(4+4)$ & $33(44.0)$ \\
\hline \multicolumn{2}{|l|}{ EAU risk category } \\
\hline Low & $0(0)$ \\
\hline Intermediate & $5(6.7)$ \\
\hline High & $70(93.3)$ \\
\hline \multicolumn{2}{|l|}{ Pathologic T stage } \\
\hline$\leq 1 \mathrm{c}$ & $0(0)$ \\
\hline $2 a-2 c$ & $33(44.0)$ \\
\hline$\geq 3 a$ & $42(56.0)$ \\
\hline \multicolumn{2}{|l|}{$\mathrm{RP}$ Gleason score } \\
\hline$\leq 6(3+3)$ & $0(0)$ \\
\hline $7(3+4)$ & $18(24)$ \\
\hline $7(4+3)$ & $25(33.3)$ \\
\hline$\geq 8(4+4)$ & $32(42.7)$ \\
\hline Median tumor volume, cc (range) & $4.43(0.32-23.96)$ \\
\hline Median tumor volume, \% (range) & $9.69(0.38-37)$ \\
\hline \multicolumn{2}{|l|}{ pN status } \\
\hline Negative & $38(50.7)$ \\
\hline Positive & 37 (49.3) \\
\hline \multicolumn{2}{|l|}{ Surgical margin status } \\
\hline Negative & $59(78.7)$ \\
\hline Positive & $16(21.3)$ \\
\hline \multicolumn{2}{|l|}{ Seminal vesicle invasion } \\
\hline Absent & $59(78.7)$ \\
\hline Present & $16(21.3)$ \\
\hline \multicolumn{2}{|l|}{ Capsular invasion } \\
\hline Absent & $33(44)$ \\
\hline Present & $42(56)$ \\
\hline \multicolumn{2}{|l|}{ Extraprostatic extension } \\
\hline Absent & $37(49.3)$ \\
\hline Present & $38(50.7)$ \\
\hline \multicolumn{2}{|l|}{ Vascular invasion } \\
\hline Absent & $64(85.3)$ \\
\hline Present & $11(14.7)$ \\
\hline \multicolumn{2}{|l|}{ Perineural invasion } \\
\hline Absent & $4(5.3)$ \\
\hline Present & $71(94.7)$ \\
\hline Biochemical recurrence & $16(21.3)$ \\
\hline Median follow-up, years (range) & $5.2(0.01-7.3)$ \\
\hline
\end{tabular}

Data are expressed as $n(\%)$ unless otherwise indicated.

EAU, European Association of Urology; pN, pathologic nodal status; PSA, prostate-specific antigen; RP, radical prostatectomy.

[including biochemical recurrence (BCR)] were available (Table 1).

The tissue microarray was built by the Oxford Centre for Histopathology Research, Oxford University Hospitals
National Health Service Foundation Trust (Oxford, UK) under the approval of the Oxford Radcliffe Biobank Ethics Committee (reference number 09/H0606/5+5). The study itself had full approval from the local ethics committee of KU Leuven (Leuven, Belgium) (reference number S55726).

\section{Immunohistochemistry}

Slides were deparaffinized and hydrated, heat-induced antigen retrieval with citrate buffer was performed, and endogenous peroxidases were blocked with $0.3 \%$ hydrogen peroxide before a serum-free protein block (X0909; Dako/ Agilent Technologies, Santa Clara, CA). Slides were incubated overnight at $4{ }^{\circ} \mathrm{C}$ with antibodies (1:50, unless stated otherwise) against SHH (ab53281; Abcam, Cambridge, UK), PTCH1 (1:300, sc-6147; Santa Cruz Biotechnology, Dallas, TX), SMO (1:100, ab72130; Abcam), SUFU (1:100, sc28847; Santa Cruz Biotechnology), GLI1 (sc-20687; Santa Cruz Biotechnology), GLI2 (1:1000, 600-401-845; Rockland, Limerick, PA), GLI3 (AF3690; R\&D Systems, Minneapolis, $\mathrm{MN}$ ), snail family transcriptional repressor 1 (SNAIL; AF3639; R\&D Systems), snail family transcriptional repressor 3 (SNAI3; NBP1-90661; Novus Biologicals, Littleton, CO), cyclin D1 (M364229; Dako/Agilent Technologies), or CD31 (IR610 Clone JC70A; Dako/Agilent Technologies). Secondary antibodies (ImmPRESS REAGENT Anti-Rabbit Ig peroxidase MP-7401, Anti-Rat Ig peroxidase MP-7404, Anti-Mouse Ig peroxidase MP-7402, or Anti-Goat Ig peroxidase MP-7405; Vector Laboratories, Burlingame, CA) were added, followed by $3.3^{\prime}$-diaminobenzidine substrate (ImmPACT DAB, SK-4105; Vector Laboratories) and counterstained with hematoxylin. Positive control specimens from patients who underwent a transurethral resection of the prostate were used to validate the specificity of the primary antibodies, which was histopathologically assessed on the basis of the expected subcellular localization [based on previous validations of these antibodies, as reported elsewhere, ${ }^{24-27}$ and/or as observed in The

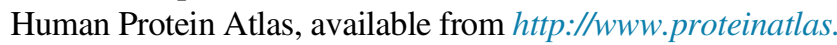
org (GLI1, GLI2, SNAI3; last accessed October 13, 2017)] and lack of nonspecific background staining. Blood vessel staining was used as an internal positive control for the validation of the anti-Snail antibody. Negative controls were performed without addition of any primary antibody to exclude nonspecific staining of secondary antibodies.

\section{Immunohistochemistry Scoring}

Immunohistochemistry staining of SHH, PTCH1, SMO, SUFU, GLI1, GLI2, GLI3, SNAIL, SNAI3, and cyclin D1 was independently scored by researchers (S.I., A.G., and/or E.L.) blinded to clinicopathological and outcome data. Potentially discordant scores were resolved by joint review of the corresponding cores. The percentage of cell immunoreactivity ( $0 \%$ to $100 \%)$ and the staining intensity (score 0 to 3 , with 0 indicating negative; 1 , weak; 2 , moderate; and 
Table 2 Differential Expression of Hh Pathway Proteins between Malignant and Benign Prostate Tissue

\begin{tabular}{|c|c|c|c|c|}
\hline \multirow[b]{2}{*}{$\begin{array}{l}\text { Paired } \\
t \text {-test }\end{array}$} & \multicolumn{2}{|c|}{ Epithelial expression } & \multicolumn{2}{|c|}{ Stromal expression } \\
\hline & $\begin{array}{l}\text { Mean difference } \\
\text { (HS tumor - } \\
\text { HS benign) }\end{array}$ & $P$ value & $\begin{array}{l}\text { Mean difference } \\
\text { (HS tumor - } \\
\text { HS benign) }\end{array}$ & $P$ value \\
\hline $\mathrm{SHH}$ & -0.099 & 0.302 & -0.340 & $<0.001^{*}$ \\
\hline PTCH1 & -0.690 & $<0.001^{*}$ & -0.321 & $<0.001^{*}$ \\
\hline SMO & 0.280 & $0.005^{*}$ & -0.120 & 0.188 \\
\hline SUFU & -0.056 & 0.58 & -0.258 & 0.004 * \\
\hline GLI1 & 0.197 & $0.041^{*}$ & -0.300 & $<0.001^{*}$ \\
\hline GLI2 & -0.221 & $0.029^{*}$ & -0.338 & $<0.001^{*}$ \\
\hline GLI3 & 0.015 & 0.827 & -0.210 & $<0.001^{*}$ \\
\hline Cyclin D1 & 0.915 & $<0.001^{*}$ & NA & NA \\
\hline SNAIL & 0.206 & $0.027^{*}$ & NA & NA \\
\hline SNAI3 & 0.565 & $<0.001^{*}$ & -0.192 & 0.009 * \\
\hline
\end{tabular}

$* P<0.05$

GLI, glioma-associated oncogene; Hh, Hedgehog; HS, histoscore; NA, not applicable (no stromal staining detected); PTCH, Patched; SHH, Sonic Hh; SM0, Smoothened; SNAI3, snail family transcriptional repressor 3; SNAIL, snail family transcriptional repressor 1 ; SUFU, suppressor of fused.

3 , strong) were recorded for each protein for both epithelial and stromal cells. Histoscores (HSs) (0 to 3 ) were calculated as the product of the percentage immunoreactivity and the staining intensity. In the malignant cores, only tumor glands were scored. If multiple staining intensities were observed in a single core, then the average HS of the whole core was recorded. For some statistical analysis, binary HSs were used (low HS was defined as $<1.5$, and high HS was defined as $\geq 1.5$, with 1.5 representing the mean HS). The subcellular localization of each protein (membranous, cytoplasmic, and/or nuclear expression) was also recorded. Microvessel density was determined using CD31 staining and counting of blood vessels per tissue core.

\section{Statistical Analysis}

The Pearson correlation coefficient was used to evaluate potential correlations between expression levels of different Hh proteins. Any dependency between binary Hh expression level and clinical subgroup was determined using a Fisher exact test or Pearson $\chi^{2}$ test. Intratumoral variation was assessed as the SD of the protein expression level within different tumor cores from each patient, and potential relationships with clinicopathological factors were investigated using a two-sample independent $t$-test. A paired $t$-test was used to investigate potential differences between protein levels in the malignant and benign cores.

Time to BCR after RP is defined as the time interval between surgery and an increase in prostate-specific antigen level to $>0.2 \mathrm{ng} / \mathrm{mL}$ (where this occurred within the cohort). Relationships between BCR and protein expression were investigated using Kaplan-Meier analysis. The influence of different risk factors on time to BCR was estimated by a log-rank test based on the binary HS of the studied proteins and clinicopathological PCa factors, including pretreatment prostate-specific antigen level, GS, clinical and pathologic T stage, lymph node involvement, and surgical margin status. A multivariate Cox proportional hazard regression model was used to determine the relative risk of BCR for these factors. Differences were considered statistically significant at $P<0.05$. SPSS Statistics version 23/24 (IBM, Armonk, NY) was used for statistical analyses. The original data sets used during this study are available as Supplemental Tables $\mathrm{S} 1$ and S2.

\section{Results}

\section{Patient Cohort}

Patient and tumor characteristics are shown in Table 1. The patient cohort predominantly comprised high-risk PCa patients $(93.3 \%)$ based on the latest European Association of Urology risk classification system [ie, prostate-specific antigen level $>20 \mathrm{ng} / \mathrm{mL}$ or biopsy GS $>7$ or clinical T (cT) stage $\geq \mathrm{cT} 2 \mathrm{c}]$. A total of $73.3 \%$ of patients had $\geq \mathrm{cT} 3 \mathrm{a}$ disease, and $44 \%$ of patients had biopsy GS $\geq 8$ disease. Pathologic analysis of RP specimens revealed that $56 \%$ of patients had $\geq \mathrm{pT} 3 \mathrm{a}$ disease and $42 \%$ had a pathologic GS score $\geq 8$. Almost half of the patients had positive lymph nodes. One in five patients developed BCR during a median follow-up of 5.2 (range, 0 to 7.3 ) years.

\section{Differential Expression of $\mathrm{Hh}$ Proteins in Benign and Malignant Prostate Tissue}

Protein expression of the primary Hh signaling components was compared between benign and malignant prostate tissue (Table 2). Representative examples of low and high protein expression of studied proteins are shown in Figure 2. Significantly higher expression levels of SMO $(P=0.005)$ and GLI1 $(P=0.041)$ were observed in malignant epithelium compared with benign prostate epithelium. Epithelial PTCH1 $(P<0.001)$ and GLI2 $(P=0.029)$ expression was significantly lower in malignant prostate epithelium than benign prostate epithelium.

These results suggest that $\mathrm{Hh}$ signaling activity is higher in malignant epithelium than benign epithelium. In accordance with this possibility, cyclin D1 $(P<0.001)$, SNAIL $(P=0.027)$, and SNAI3 $(P<0.001)$, which are known target genes of $\mathrm{Hh}$ signaling and involved in cell cycle progression and epithelial-to-mesenchymal transition, respectively, were more highly expressed in tumor cells than benign epithelium (Table 2). Lower expression of multiple Hh components (SHH, PTCH1, SUFU, GLI1, GLI2, and GLI3; $P<0.005$ for each) was observed in the stroma of $\mathrm{PCa}$ tissue compared with stroma in surrounding benign tissue. In addition, protein expression of SNAI3 $(P=0.009)$ was lower in tumor-associated stroma than benign tissue stroma. Microvessel density was higher in 

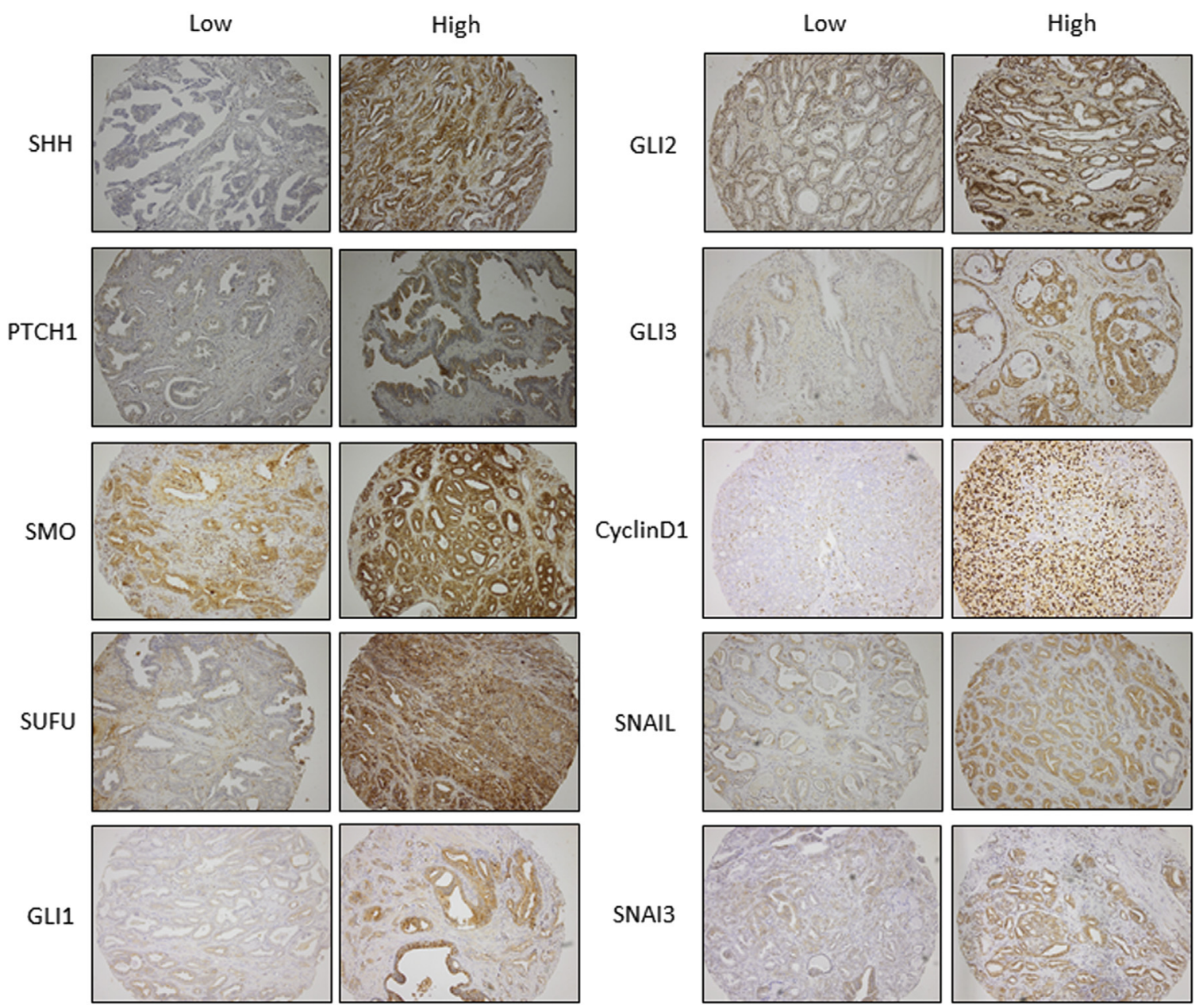

Figure 2 Representative images of low and high hedgehog pathway protein expression in malignant prostate tissue cores. Tissue cores were stained for Sonic hedgehog (SHH), Patched 1 (PTCH1), Smoothened (SM0), suppressor of fused (SUFU), glioma-associated oncogene (GLI) 1, GLI2, GLI3, cyclin D1, snail family transcriptional repressor 1 (SNAIL), and snail family transcriptional repressor 3 (SNAI3). Original magnification, $\times 100$.

malignant prostate tissue compared with benign prostate tissue $(P<0.001)$. Taken together, these results suggest that Hh signaling activity is higher in malignant prostate epithelium than in surrounding tumor-associated stroma and that autocrine Hh signaling may play a role in PCa.

\section{Correlations in Expression between Different Hh Proteins}

Potential relationships among differential expression of $\mathrm{Hh}$ signaling proteins were evaluated in benign and malignant prostate tissue using a Pearson correlation. Significant correlations were observed between Hh proteins (Table 3), with strong correlations being defined as a correlation coefficient $\geq 0.5$. In malignant epithelium, SHH was coexpressed with SMO $(r=0.52)$ and GLI1 $(r=0.54)$, these being important activators of $\mathrm{Hh}$ signaling. In addition, SMO expression was coexpressed with SNAI3 $(r=0.58)$ in malignant epithelium. In malignant stroma, SMO was strongly expressed with GLI3 $(r=0.65)$, SNAI3 $(r=0.53)$, and SHH $(r=0.61)$. Furthermore, stromal SMO expression was associated with epithelial SNAIL expression $(r=0.59)$.

In benign prostate epithelium, SMO expression was correlated with GLI3 $(r=0.53)$, SNAI3 $(r=0.57)$, and GLI2 $(r=0.70)$ expression. Stromal and epithelial SNAI3 expression was also correlated $(r=0.67)$. In benign stroma, SMO expression was correlated with GLI3 $(r=0.59)$ and SHH $(r=0.60)$ expression, and a correlation was seen between GLI3 and SHH expression $(r=0.57)$.

\section{GLI1 Expression in the Tumor Epithelium Correlates with Vascular Invasion and Higher Pathologic Gleason Score}

Potential correlations between expression of $\mathrm{Hh}$ proteins and known clinicopathological factors were investigated. Increased total GLI1 expression in malignant prostate epithelium was observed to correlate with an increased number of blood vessels $(P=0.046)$. A distinction was 
Table 3 Correlations between Hh Proteins in the Epithelial and Stromal Cells in the Tumor and Benign Prostate Tissue

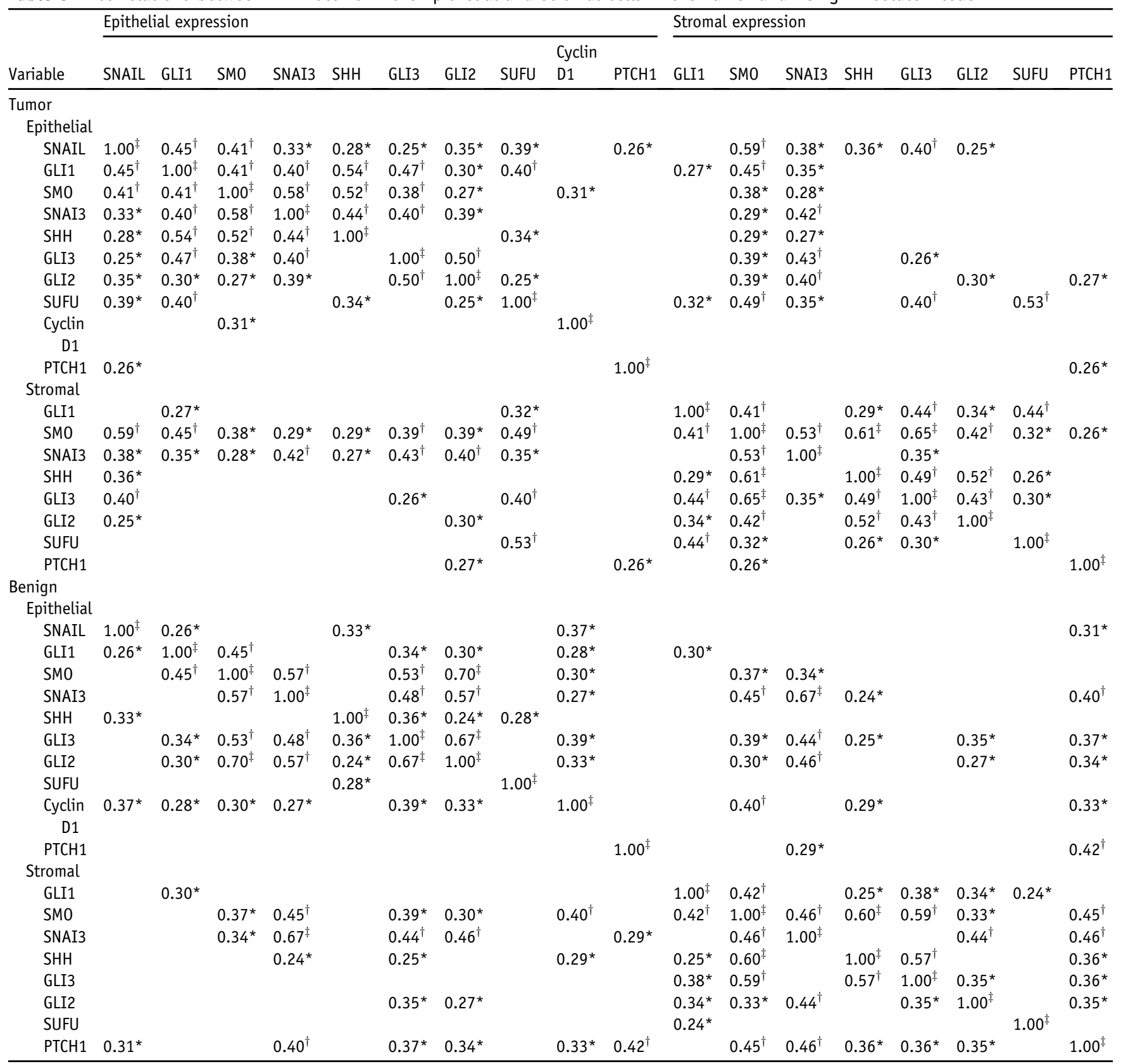

Pearson correlation coefficients of statistically significant $(P<0.05)$ correlations are given. Empty cells indicate that there was no significant result.

*Weak correlation.

${ }^{\dagger}$ Moderate correlation.

${ }^{\ddagger}$ Strong correlation.

GLI, glioma-associated oncogene; Hh, Hedgehog; PTCH, Patched; SHH, Sonic Hh; SM0, Smoothened; SNAI3, snail family transcriptional repressor 3; SNAIL, snail family transcriptional repressor 1; SUFU, suppressor of fused.

then made between nuclear GLI protein expression (irrespective of cytoplasmic expression) and sole cytoplasmic expression, to investigate active and inactive Hh signaling. In malignant prostate cells with positive nuclear GLI1 expression, higher GLI1 expression was observed to correlate with a higher pathologic GS $(\mathrm{GS}>7)(P=0.041)$ (Table 4 and Supplemental Figure S1).

\section{Intratumor Heterogeneity}

The expression of each Hh pathway protein in each of four tumor cores from each individual patient was evaluated to investigate potential intratumor heterogeneity ${ }^{28}$ and to assess whether any observed heterogeneity may relate to a particular patient subgroup (Supplemental Table S3). SDs of the Hh 
Table 4 Correlation between GLI1 Expression and Clinicopathological Factors

\begin{tabular}{|c|c|c|c|c|c|c|c|c|c|}
\hline \multirow[b]{3}{*}{ Fisher exact test } & \multicolumn{3}{|c|}{ Nuclear GLI1 } & \multicolumn{3}{|c|}{ Cytoplasmic GLI1 } & \multicolumn{3}{|c|}{ Total GLI1 } \\
\hline & \multicolumn{2}{|l|}{$n$} & \multirow[b]{2}{*}{$P$ value } & \multicolumn{2}{|l|}{$n$} & \multirow[b]{2}{*}{$P$ value } & \multicolumn{2}{|l|}{$n$} & \multirow[b]{2}{*}{$P$ value } \\
\hline & Low & High & & Low & High & & Low & High & \\
\hline $\mathrm{PSA} \leq 10 \mathrm{ng} / \mathrm{mL}$ & 20 & 6 & & 2 & 4 & & 22 & 10 & \\
\hline $\mathrm{PSA}>10 \mathrm{ng} / \mathrm{mL}$ & 20 & 12 & & 3 & 3 & & 23 & 15 & \\
\hline Clinical T stage & & & 0.342 & & & 0.417 & & & 0.098 \\
\hline CT $>2$ & 27 & 15 & & 4 & 7 & & 31 & 22 & \\
\hline Biopsy Gleason score & & & 0.565 & & & 1.000 & & & 0.454 \\
\hline $\mathrm{GS} \leq 7$ & 26 & 10 & & 2 & 3 & & 28 & 13 & \\
\hline $\mathrm{GS}>7$ & 14 & 8 & & 3 & 4 & & 17 & 12 & \\
\hline Pathologic T stage & & & 0.571 & & & 1.000 & & & 0.313 \\
\hline $\mathrm{pT} \leq 2$ & 20 & 7 & & 1 & 1 & & 21 & 8 & \\
\hline pN stage & & & 0.682 & & & 0.472 & & & 0.261 \\
\hline Negative & 22 & 9 & & 1 & 1 & & 23 & 22 & \\
\hline Positive & 18 & 9 & & 4 & 6 & & 10 & 15 & \\
\hline Surgical margin status & & & 0.689 & & & 0.372 & & & 0.241 \\
\hline Negative & 34 & 14 & & 3 & 4 & & 37 & 18 & \\
\hline Positive & 6 & 4 & & 2 & 3 & & 8 & 7 & \\
\hline Seminal vesicle invasion & & & 0.483 & & & 1.000 & & & 0.370 \\
\hline Negative & 36 & 14 & & 3 & 4 & & 37 & 18 & \\
\hline Positive & 6 & 4 & & 2 & 3 & & 8 & 7 & \\
\hline Capsular invasion & & & 1.000 & & & 0.417 & & & 0.614 \\
\hline Negative & 19 & 9 & & 1 & 0 & & 20 & 9 & \\
\hline Perineural invasion & & & 0.528 & & & & & & 1.000 \\
\hline Negative & 1 & 1 & & 5 & 7 & & 1 & 1 & \\
\hline Positive & 39 & 17 & & 5 & 7 & & 44 & 24 & \\
\hline
\end{tabular}

$* P<0.05$.

GLI, glioma-associated oncogene; GS, Gleason sum score; pN, pathologic nodal status; PSA, prostate-specific antigen; RP, radical prostatectomy.

pathway proteins were compared with different patient subgroups to investigate whether heterogeneous expression patterns may be associated with clinicopathological features. Less intratumor variation of epithelial SUFU expression was observed in patients with a high versus low biopsy GS $(P=0.026)$. Patients with positive lymph node metastases demonstrated more homogeneous PTCH1 expression $(P=0.013)$ within the primary tumor epithelium. Stromal SHH expression was less variable in patients with a high biopsy GS $(P=0.009)$, high pathologic GS $(P=0.016)$, or positive lymph node metastases $(P=0.027)$. Epithelial expression of SNAIL $(P=0.036)$ and stromal expression of SMO $(P=0.029)$ and GLI3 $(P=0.038)$ were more homogeneous in PCa cases with capsular invasion (stage $\geq$ pT3) than in cases of $\leq \mathrm{pT} 2$ stage disease. Taken together, these data suggest that patients with a more aggressive $\mathrm{PCa}$ phenotype have a more consistent (ie, homogeneous) staining pattern of several important Hh pathway proteins.

Higher Epithelial PTCH1 Expression Correlates with Unfavorable Patient Outcome

Univariate analysis revealed that higher epithelial expression of PTCH1 in malignant prostate cells correlated with a shorter time to BCR (Figure 3 and Supplemental Table S4). This observation was also seen in a multivariate analysis (Table 5), indicating that epithelial PTCH1 expression in malignant tissue may be a prognostic marker for BCR. 


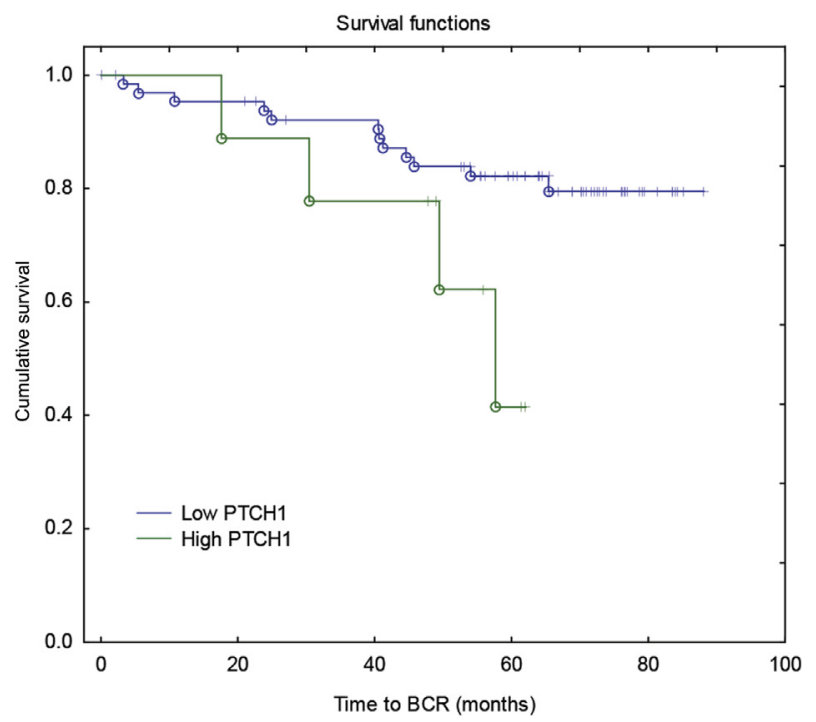

Figure 3 Correlations between epithelial Patched 1 (PTCH1) and patient outcome. Kaplan-Meier analysis of time to biochemical relapse (BCR) for prostate cancer patients with high (green) or low (blue) epithelial PTCH1 expression in the tumor (log-rank test: $\chi^{2}=7.728, P=0.005$ ). Low and high PTCH1 expression levels are defined as such based on binary histoscores of $<1.5$ and $\geq 1.5$, respectively.

Tumor volume was also observed to be an independent predictor for BCR.

\section{Discussion}

Increasing evidence suggests a role for active Hh signaling in the development and progression of PCa to an advanced phenotype. Although Hh signaling plays an essential role in the embryonic development of the prostate, its role in the adult prostate is usually limited. In $\mathrm{PCa}$, uncontrolled reactivation of $\mathrm{Hh}$ signaling appears to occur, which is more pronounced in the advanced stages of this malignancy. A potential relationship between Hh signaling and androgenindependent $\mathrm{PCa}$ has been described by several research groups. Moreover, preclinical data demonstrate that inhibition of Hh signaling may reduce prostate cancer invasiveness and metastatic potential. ${ }^{8}$

In this study, the expression profile of the principal $\mathrm{Hh}$ signaling pathway components was determined in a cohort of predominantly high-risk $\mathrm{PCa}$ patients treated by RP. The management of high-risk $\mathrm{PCa}$ is one of the most important challenges in contemporary clinical practice because of the large variation in treatment response observed in this group. Although many high-risk PCa patients have a good prognosis after RP performed with curative intent, unfortunately a significant number of patients still succumb to recurrent and progressive disease, with potentially lethal consequences. ${ }^{29}$ There is therefore an unmet clinical need for further stratification tools, such as robust biological markers of potentially aggressive disease, within this specific high-risk cohort, such that these biomarkers might be used in routine clinical practice to improve patient outcomes. ${ }^{5,13}$ We hypothesized that increased $\mathrm{Hh}$ signaling pathway activity may represent a promising biomarker of increased disease aggressiveness in high-risk PCa patients receiving RP with curative intent.

The expression levels of $\mathrm{Hh}$ signaling proteins differ between benign and malignant prostate tissue. Higher expression of the Hh pathway activators SMO and GLI1, and lower expression of the negative regulator PTCH1, was observed in this high-risk PCa cohort. GLI2 expression was also reduced in malignant versus benign prostate epithelium. Depending on the cellular context, GLI2 is processed to a truncated (GLI2-78) repressor form, ${ }^{30}$ but little is known about the specific mechanisms that trigger this processing. It is known that when the Hh pathway is inactive, with no ligand bound to PTCH1, the GLI transcription factors are phosphorylated by protein kinase A, casein kinase 1 , and glycogen synthase kinase $3 \beta$, which results in targeting of GLI proteins to the proteasome for degradation. ${ }^{30}$ Fulllength GLI2 (GLI2-185) and truncated GLI2 (GLI2-78) could not be discriminated; therefore, clear conclusions cannot be made regarding the exact function of GLI2 in this setting. Nevertheless, the observations that both GLI1 and SMO appear to be up-regulated in the tumor, whereas PTCH1 is down-regulated, suggest that the repressor form of GLI2 is also down-regulated in PCa, indicating that $\mathrm{Hh}$ signaling activity is higher in PCa than in benign prostate epithelium. In accordance with this possibility, epithelial expression of cyclin D1, SNAIL, and SNAI3 was higher in malignant than in benign epithelium, consistent with greater Hh pathway signaling and potentially resulting in enhanced proliferation and epithelial-to-mesenchymal transition. In contrast, down-regulation of $\mathrm{Hh}$ signaling activity was observed in tumor-associated stroma compared with benign stroma. This finding is consistent with that of a previous report by Tzelepi et al. ${ }^{12}$ Higher epithelial and lower stromal $\mathrm{Hh}$ expression may indicate autocrine $\mathrm{Hh}$ signaling in $\mathrm{PCa}$ tissue. Previous reports have also demonstrated an important role for autocrine $\mathrm{Hh}$ signaling in PCa. ${ }^{10,11,31,32}$ In contrast, Fan et $\mathrm{al}^{33}$ found, in a xenograft PCa model, that paracrine $\mathrm{Hh}$ signaling stimulated the tumor microenvironment to induce tumor growth by the production of growth factors. A strong correlation was observed between stromal SMO and SNAI3 expression, suggesting that paracrine Hh signaling may also be biologically relevant in PCa. Taken together, the available evidence suggests that $\mathrm{Hh}$ signaling in $\mathrm{PCa}$ is likely to be a combination of both autocrine and paracrine signaling.

Table 5 Multivariate Cox Proportional Hazard Regression Analysis of Time to BCR

\begin{tabular}{llll}
\hline Variable & Hazard ratio & $95 \%$ CI & $P$ value \\
\hline Tumor volume, cC & 1.216 & $1.079-1.371$ & $0.001^{*}$ \\
Epithelial PTCH1 & 4.977 & $1.585-15.626$ & $0.006^{*}$ \\
\hline
\end{tabular}

${ }^{*} P<0.05$.

$\mathrm{BCR}$, biochemical relapse; PTCH, Patched. 
Nuclear GLI1 expression was positively correlated with pathologic GS, indicating that this may be a marker of an aggressive tumor phenotype. This accords with a study by Kim et al, ${ }^{34}$ in which a correlation was observed between GLI1 and GS. Although Kim et $\mathrm{al}^{34}$ observed that expression of other Hh proteins (SHH, PTCH1, and SMO) also correlated with GS, this finding was not observed in our study. One reason for this might be that in this study most of the patients had high-risk PCa, whereas the study by Kim et $\mathrm{al}^{34}$ included patients with low-, intermediate-, and highrisk PCa. Kim et $\mathrm{al}^{34}$ also observed that epithelial SHH in the tumor was an independent prognostic marker for BCR, whereas in this study epithelial PTCH1 expression was associated with an unfavorable patient outcome.

A significant association was observed between epithelial PTCH1 expression and BCR in this predominantly high-risk PCa cohort. Although this finding may appear to contradict the known function of PTCH1 as a negative regulator of $\mathrm{Hh}$ signaling, PTCH1 activity is dependent on its precise subcellular localization. Membranous PTCH1 localization is associated with an inhibitory function, whereas nuclear and cytoplasmic PTCH1 localization indicates active $\mathrm{Hh}$ signaling. PTCH1 expression was found to be mostly present in the cytoplasm of malignant epithelium, and this particular localization is indicative of active Hh signaling.

There are inherent limitations in our study attributable to its retrospective nature and the use of immunohistochemistry. Furthermore, for each patient, all tumor cores $(n=4)$ were taken from the same macroblock, and for some patients, not all cores could be evaluated, which might compromise a detailed analysis of intratumor heterogeneity. Also, because the number of patients $(n=75)$ in this study is modest, the data should be validated in a larger patient cohort.

In conclusion, active Hh signaling appears to be greater in malignant prostate epithelium than in benign prostate epithelium. In addition, the available evidence suggests that both autocrine and paracrine Hh signaling may play roles in PCa progression, and GLI1 expression might represent a clinically useful biomarker of tumor aggressiveness in highrisk PCa treated with RP. PTCH1 may also be a good candidate for a clinically useful biomarker of outcome in high-risk PCa patients treated with RP; however, this requires validation in larger studies.

\section{Supplemental Data}

Supplemental material for this article can be found at https://doi.org/10.1016/j.ajpath.2017.11.019.

\section{References}

1. Torre LA, Bray F, Siegel RL, Ferlay J, Lortet-Tieulent J, Jemal A: Global cancer statistics, 2012. CA Cancer J Clin 2015, 65:87-108

2. D'Amico AV, Cote K, Loffredo M, Renshaw AA, Chen MH: Pretreatment predictors of time to cancer specific death after prostate specific antigen failure. J Urol 2003, 169:1320-1324
3. Mottet N, Bellmunt J, Bolla M, Briers E, Cumberbatch MG, De SM, Fossati N, Gross T, Henry AM, Joniau S, Lam TB, Mason MD, Matveev VB, Moldovan PC, van den Bergh RC, Van den Broeck T, van der Poel HG, van der Kwast TH, Rouviere O, Schoots IG, Wiegel T, Cornford P: EAU-ESTRO-SIOG guidelines on prostate cancer, part 1: screening, diagnosis, and local treatment with curative intent. Eur Urol 2017, 71:618-629

4. D'Amico AV, Moul J, Carroll PR, Sun L, Lubeck D, Chen MH: Cancer-specific mortality after surgery or radiation for patients with clinically localized prostate cancer managed during the prostatespecific antigen era. J Clin Oncol 2003, 21:2163-2172

5. Joniau S, Briganti A, Gontero P, Gandaglia G, Tosco L, Fieuws S, Tombal B, Marchioro G, Walz J, Kneitz B, Bader P, Frohneberg D, Tizzani A, Graefen M, van Cangh P, Karnes RJ, Montorsi F, Van Poppel H, Spahn M; European Multicenter Prostate Cancer Clinical and Translational Research Group (EMPaCT): Stratification of highrisk prostate cancer into prognostic categories: a European multiinstitutional study. Eur Urol 2015, 67:157-164

6. Azoulay S, Terry S, Chimingqi M, Sirab N, Faucon H, Gil Diez de MS, Moutereau S, Maille P, Soyeux P, Abbou C, Salomon L, Vacherot F, de La Taille A, Loric S, Allory Y: Comparative expression of Hedgehog ligands at different stages of prostate carcinoma progression. J Pathol 2008, 216:460-470

7. Efstathiou E, Karlou M, Wen S, Hoang A, Pettaway CA, Pisters LL, Maity S, Troncoso P, Logothetis CJ: Integrated Hedgehog signaling is induced following castration in human and murine prostate cancers. Prostate 2013, 73:153-161

8. Gonnissen A, Isebaert S, Haustermans K: Hedgehog signaling in prostate cancer and its therapeutic implication. Int J Mol Sci 2013, 14: 13979-14007

9. Ibuki N, Ghaffari M, Pandey M, Iu I, Fazli L, Kashiwagi M, Tojo H, Nakanishi O, Gleave ME, Cox ME: TAK-441, a novel investigational smoothened antagonist, delays castration-resistant progression in prostate cancer by disrupting paracrine hedgehog signaling. Int $\mathrm{J}$ Cancer 2013, 133:1955-1966

10. Karhadkar SS, Bova GS, Abdallah N, Dhara S, Gardner D, Maitra A, Isaacs JT, Berman DM, Beachy PA: Hedgehog signalling in prostate regeneration, neoplasia and metastasis. Nature 2004, 431:707-712

11. Sheng T, Li C, Zhang X, Chi S, He N, Chen K, McCormick F, Gatalica Z, Xie J: Activation of the hedgehog pathway in advanced prostate cancer. Mol Cancer 2004, 3:29

12. Tzelepi V, Karlou M, Wen S, Hoang A, Logothetis C, Troncoso P, Efstathiou E: Expression of hedgehog pathway components in prostate carcinoma microenvironment: shifting the balance towards autocrine signalling. Histopathology 2011, 58:1037-1047

13. Yossepowitch O, Eggener SE, Serio AM, Carver BS, Bianco FJ Jr, Scardino PT, Eastham JA: Secondary therapy, metastatic progression, and cancer-specific mortality in men with clinically high-risk prostate cancer treated with radical prostatectomy. Eur Urol 2008, 53:950-959

14. Humke EW, Dorn KV, Milenkovic L, Scott MP, Rohatgi R: The output of Hedgehog signaling is controlled by the dynamic association between Suppressor of Fused and the Gli proteins. Genes Dev 2010, 24:670-682

15. Kogerman $\mathrm{P}$, Grimm $\mathrm{T}$, Kogerman $\mathrm{L}$, Krause $\mathrm{D}$, Unden $\mathrm{AB}$ Sandstedt B, Toftgard R, Zaphiropoulos PG: Mammalian suppressorof-fused modulates nuclear-cytoplasmic shuttling of Gli-1. Nat Cell Biol 1999, 1:312-319

16. Marigo V, Johnson RL, Vortkamp A, Tabin CJ: Sonic hedgehog differentially regulates expression of GLI and GLI3 during limb development. Dev Biol 1996, 180:273-283

17. Teglund S, Toftgard R: Hedgehog beyond medulloblastoma and basal cell carcinoma. Biochim Biophys Acta 2010, 1805:181-208

18. Wang B, Fallon JF, Beachy PA: Hedgehog-regulated processing of Gli3 produces an anterior/posterior repressor gradient in the developing vertebrate limb. Cell 2000, 100:423-434 
19. Yang L, Xie G, Fan Q, Xie J: Activation of the hedgehog-signaling pathway in human cancer and the clinical implications. Oncogene 2010, 29:469-481

20. Dai P, Akimaru H, Tanaka Y, Maekawa T, Nakafuku M, Ishii S: Sonic Hedgehog-induced activation of the Glil promoter is mediated by GLI3. J Biol Chem 1999, 274:8143-8152

21. Iwasaki H, Nakano K, Shinkai K, Kunisawa Y, Hirahashi M, Oda Y, Onishi H, Katano M: Hedgehog Gli3 activator signal augments tumorigenicity of colorectal cancer via upregulation of adherencerelated genes. Cancer Sci 2013, 104:328-336

22. Trnski D, Sabol M, Gojevic A, Martinic M, Ozretic P, Musani V, Ramic S, Levanat S: GSK3beta and Gli3 play a role in activation of Hedgehog-Gli pathway in human colon cancer: targeting GSK3beta downregulates the signaling pathway and reduces cell proliferation. Biochim Biophys Acta 2015, 1852:2574-2584

23. Van den Bergh L, Lerut E, Haustermans K, Deroose CM, Oyen R, Isebaert S, Budiharto T, Ameye F, Mottaghy FM, Bogaerts K, Van Poppel H, Joniau S: Final analysis of a prospective trial on functional imaging for nodal staging in patients with prostate cancer at high risk for lymph node involvement. Urol Oncol 2015, 33:109-131

24. Verrill C, Cerundolo L, Mckee C, White M, Kartsonaki C, Fryer E, Morris E, Brewster S, Ratnayaka I, Marsden L, Lilja H, Muschel R, Lu X, Hamdy F, Bryant RJ: Altered expression of epithelial-tomesenchymal transition proteins in extraprostatic prostate cancer. Oncotarget 2016, 7:1107-1119

25. Cigna N, Farrokhi Moshai E, Brayer S, Marchal-Somme J, WémeauStervinou L, Fabre A, Mal H, Lesèche G, Dehoux M, Soler P, Crestani B, Mailleux AA: The hedgehog system machinery controls transforming growth factor- $\beta$-dependent myofibroblastic differentiation in humans: involvement in idiopathic pulmonary fibrosis. Am J Pathol 2012, 181:2126-2137

26. Maréchal R, Bachet JB, Calomme A, Demetter P, Delpero JR, Svrcek M, Cros J, Bardier-Dupas A, Puleo F, Monges G, Hammel P, Louvet C, Paye F, Bachelier P, Le Treut YP, Vaillant JC, Sauvanet A, André T, Salmon I, Devière J, Emile JF, Van Laethem JL: Sonic hedgehog and Gli1 expression predict outcome in resected pancreatic adenocarcinoma. Clin Cancer Res 2015, 21:1215-1224

27. Yoshizaki A, Nakayama T, Naito S, Wen CY, Sekine I: Expressions of sonic hedgehog, patched, smoothened and Gli-1 in human intestinal stromal tumors and their correlation with prognosis. World J Gastroenterol 2006, 12:5687-5691

28. Goethals L, Perneel C, Debucquoy A, De SH, Borghys D, Ectors N, Geboes K, McBride WH, Haustermans KM: A new approach to the validation of tissue microarrays. J Pathol 2006, 208:607-614

29. Briganti A, Joniau S, Gontero P, Abdollah F, Passoni NM, Tombal B, Marchioro G, Kneitz B, Walz J, Frohneberg D, Bangma CH, Graefen M, Tizzani A, Frea B, Karnes RJ, Montorsi F, Van Poppel H, Spahn M: Identifying the best candidate for radical prostatectomy among patients with high-risk prostate cancer. Eur Urol 2012, 61:584-592

30. Pan Y, Bai CB, Joyner AL, Wang B: Sonic hedgehog signaling regulates Gli2 transcriptional activity by suppressing its processing and degradation. Mol Cell Biol 2006, 26:3365-3377

31. McKee CM, Xu D, Cao Y, Kabraji S, Allen D, Kersemans V, Beech J, Smart S, Hamdy F, Ishkanian A, Sykes J, Pintile M, Milosevic M, van der Kwast T, Zafarana G, Ramnarine VR, Jurisica I, Mallof C, Lam W, Bristow RG, Muschel RJ: Protease nexin 1 inhibits hedgehog signaling in prostate adenocarcinoma. J Clin Invest 2012, 122:4025-4036

32. Sanchez P, Hernandez AM, Stecca B, Kahler AJ, DeGueme AM, Barrett A, Beyna M, Datta MW, Datta S, Altaba A: Inhibition of prostate cancer proliferation by interference with SONIC HEDGEHOG-GLI1 signaling. Proc Natl Acad Sci U S A 2004, 101: $12561-12566$

33. Fan L, Pepicelli CV, Dibble CC, Catbagan W, Zarycki JL, Laciak R, Gipp J, Shaw A, Lamm ML, Munoz A, Lipinski R, Thrasher JB, Bushman W: Hedgehog signaling promotes prostate xenograft tumor growth. Endocrinology 2004, 145:3961-3970

34. Kim TJ, Lee JY, Hwang TK, Kang CS, Choi YJ: Hedgehog signaling protein expression and its association with prognostic parameters in prostate cancer: a retrospective study from the view point of new 2010 anatomic stage/prognostic groups. J Surg Oncol 2011, 104:472-479 